\title{
Bowel management outcomes in individuals with long-term spinal cord injuries
}

\author{
Robert Menter ${ }^{1}$, David Weitzenkamp ${ }^{1}$, Dianne Cooper ${ }^{2}$, Jenny Bingley ${ }^{3}$, Susan Charlifue ${ }^{1}$ and Gale Whiteneck ${ }^{1}$ \\ ${ }^{1}$ Craig Hospital, Englewood, Colorado, USA; ${ }^{2}$ National Spinal Injuries Centre, Stoke Mandeville Hospital, \\ Aylesbury, Buckinghamshire, England; ${ }^{3}$ Regional Spinal Injuries Centre, District General Hospital, Southport, \\ Merseyside, England
}

\begin{abstract}
Outcomes of bowel management were evaluated in 1993 among 221 British long-term spinal cord injury survivors who were followed-up as part of a longitudinal study of aging with spinal cord injury. Representing a population-based sample followed up by two regional spinal cord injury treatment centres - Stoke Mandeville Hospital in Aylesbury and the District General Hospital in Southport, England - all had been injured at least 20 years when the study was begun in 1990; all underwent comprehensive assessments and extensive interviewing at their centres in 1990 and 1993. When assessed in 1993, 42\% of the entire sample reported constipation, $35 \%$ reported gastrointestinal pain, and $27 \%$ complained of bowel accidents. Physician examiners diagnosed significantly more hemorrhoids among those using primarily suppositories and enemas to manage their bowels $(P<0.05)$ and more constipation among persons with paraplegia $(P<0.05)$ and those using digital stimulation, manual evacuation, or increased abdominal pressure in their bowel programs. Fecal incontinence and diarrhea was diagnosed three times more often in participants with tetraplegia as in other neurological groupings. These and other findings suggest future research needs and directions relative to long-term bowel management for spinal cord injury survivors.
\end{abstract}

Keywords: spinal cord injuries; bowel outcomes; complications

\section{Introduction}

While bowel dysfunction and acute management of the neurogenic bowel in individuals with spinal cord injury (SCI) of new onset has been well documented, ${ }^{1-11}$ long term bowel management outcomes are among the least discussed topics in the SCI literature. The frequent misperception that there is little serious morbidity or mortality associated with bowel dysfunction, coupled with the diagnostic difficulties created by SCI survivor's lack of sensation and mobility, ${ }^{9}$ may account for some of this disinterest.

However, compared with the general population, SCI is associated with increased severity and frequency of bowel problems. Much of this morbidity has been linked to effects of autonomic nervous system dysfunction $^{1-3}$ such as delayed gastric emptying, ${ }^{4}$ slowed bowel transit time, ${ }^{2,3}$ and poor colonic motility, ${ }^{5}$ and frequently manifests itself as complaints of post-prandial abdominal distention, ${ }^{6}$ constipation, ${ }^{2,5,6}$ poor colonic compliance, ${ }^{2}$ and autonomic dysreflexia. ${ }^{7}$ In one review of 127 aging veterans with spinal cord injuries, $27 \%$ had bowel complaints, including not only autonomic hyperreflexia arising from the gastrointestinal tract, but also pain,

Correspondence: R Menter abdominal distention, difficult bowel evacuation, and hemorrhoids. Twenty-three percent of the study participants had been admitted to the hospital at least once since injury for bowel-related problems, and the prevalence of problems increased with length of time injured.?

Seemingly, few other studies have tracked GI disturbances and their frequency in long-injured populations, and few have examined the impact that bowel management technique has on reported complications-despite the fact that such information could permit SCI survivors to make the changes and interventions necessary to avert more medically intensive treatment. ${ }^{9}$ This descriptive study, therefore, examines the association of bowel management techniques with bowel complications, as well as analyzing the effects of both age and duration of injury in individuals who have been spinal cord injured for 20 or more years.

\section{Methodology}

In 1990 an investigation of long-term health and psychosocial problems in an aging SCI population was undertaken in Great Britain. ${ }^{12}$ This study included individuals who had been injured for at least 20 years 
and received their rehabilitation at either the National Spinal Injuries Centre at Stoke Mandeville Hospital in Aylesbury, or at the Regional Spinal Injuries Centre at the District General Hospital in Southport. At the outset, 282 individuals participated in this extensive investigation. In 1993, 227 of these individuals participated in a second phase of the same study. All individuals underwent comprehensive evaluation using questionnaires, physical examinations, and physiologic measurements during both data collection periods. In addition, a comprehensive medical record review of the spinal centre and general practitioner records was performed to abstract all diagnoses and operations occurring in the time period between 1990 and 1993 .

This analysis focuses on 221 of the 227 individuals participating in both 1990 and 1993. Six individuals were excluded because their bowel management programs were not classifiable into one of the four categories of bowel management, described below:

(a) Bowel emptying via mechanical stimulation of the rectal reflex (digital stimulation) and/or by manual evacuation;

(b) Bowel emptying via chemical stimulation of the colorectal reflex with suppositories, including four cases of enema usage;

(c) Bowel emptying using increased intra-abdominal pressure (Valsalva) or manually-generated external pressure;

(d) Bowel emptying with no intervention required (near normal but with hyperactive anal rectal reflex).

In 34 cases participants used both mechanical and chemical stimulation. For these, the participant was assigned to the mechanical stimulation group. Finally, if individuals had changed methods, only the currently used method was recorded.

Specific terms used throughout this discussion are defined as follows:

Constipation-no bowel movement for 3 or more days, or inadequate emptying during bowel program attempt for two or more consecutive tries (by either physician diagnosis or selfreport).

Fecal incontinence-bowel movement of any consistency-including diarrhea-like stool-occurring without preparation or intent and not due to recent gastrointestinal acute illness such as influenza or food poisoning; and not attributable to recent food intake (such as spicy foods, large quantities of fruit, etc).

GI pain-self-reported gastric or abdominal discomfort which is not directly related to acute gastrointestinal illness such as influenza or food poisoning; and not attributable to recent food intake (such as spicy or 'gassy' foods).

Differences in outcomes with respect to bowel management techniques and neurological group were evaluated using chi-square techniques. For the latter, three separate groups - paraplegia, ASIA Impairment $\mathrm{A}, \mathrm{B}$, or $\mathrm{C}$; tetraplegia $\mathrm{A}, \mathrm{B}$, or $\mathrm{C}$; and all ASIA
Impairment Ds - were identified. ${ }^{12}$ Previous analyses suggest the usefulness of this division, as members of each group share similar functional abilities and support needs. ${ }^{13,14}$ The effects of medication use were also evaluated. Further analysis, also using chi-square tests, was performed to detect which, if any, bowel management technique seemed to have a statistically significant different frequency of complications than did all other techniques combined. Finally, logistic regression was used to evaluate the relative effects of age and duration of injury on the outcome variables.

\section{Results}

Sixty-five individuals $(29 \%)$ in the study group had Tetraplegia, ASIA Impairment ABC; ${ }^{12} 109$ (49\%) had Paraplegia $\mathrm{ABC},{ }^{12}$ and the remaining $47(21 \%)$ were classified as having very incomplete, ASIA Impairment $\mathrm{D}^{12}$ paraplegia or tetraplegia. The mean ages and duration of injury in 1993 for these groups are seen in Table 1 .

The distribution of bowel management technique for each of the three neurologic groups is illustrated in Table 2. While more than one-half of those with the most incomplete injuries (ASIA Impairment $\mathrm{D}^{12}$ ) had near-normal bowel programs, all four methods of bowel management were used by at least some members of each of the three impairment groups.

Outcomes of hemorrhoids, rectal abscesses, fecal incontinence, and constipation (based on physician diagnosis)-analyzed by method of bowel management-are reviewed in Table 3 . Those using chemical stimulation for their bowel management had significantly more diagnoses of hemorrhoids $(P<0.05)$. Although those with near-normal bowel management techniques had the lowest percentage of hemorrhoids and other complications as well - these differences were not statistically significant. These same physiciandiagnosed outcomes, analyzed by neurologic grouping, are depicted on Table 4. Fecal incontinence was significantly higher in the tetraplegia ABC group $(P=0.003)$, while constipation was significantly higher in the paraplegia ABC group $(P=0.001)$.

Self-reports of constipation, incontinence, and GI pain by bowel management technique are illustrated in Table 5. Constipation was perceived to be a problem for more than $40 \%$ of all individuals. GI pain differed significantly among the various management methods. Those using physical stimulation techniques reported GI pain more frequently than did all others

Table 1 Distribution of age and duration of injury by neurologic group

\begin{tabular}{lcc}
\hline $\begin{array}{l}\text { ASIA } \\
\text { neurologic group }\end{array}$ & Mean age & $\begin{array}{c}\text { Mean years } \\
\text { post-injury }\end{array}$ \\
\hline Tetraplegia ABC $(n=65)$ & 51.0 & 27.9 \\
Paraplegia ABC $(n=109)$ & 56.3 & 31.3 \\
All Ds $(n=47)$ & 56.6 & 29.7 \\
\hline
\end{tabular}


$(P=0.005)$, while those with chemical stimulation techniques reported the least $(P=0.029)$. The relatively small number of people using the Valsalva technique precluded any meaningful comparison of this group against all other groups. Similarly, analyses evaluating the effect of medications on bowel function were not possible because of the relative infrequency of medication usage throughout the sample: only four study participants reported taking anticholinergics as part of their bladder management programs and only six used prescription stool softeners. Table 6, which presents self-reported complications by neurological impairment group, reflects a similarly high rate of perceived constipation; however, differences in reported complications across these three groups were not significant. Additionally, when the group was

Table 2 Bowel management technique by neurologic group

\begin{tabular}{|c|c|c|c|c|c|c|}
\hline \multirow{2}{*}{$\begin{array}{l}\text { Bowel management } \\
\text { technique }\end{array}$} & \multicolumn{2}{|c|}{$\begin{array}{c}\text { Tetraplegia } \\
\qquad A B C^{12}\end{array}$} & \multicolumn{2}{|c|}{$\begin{array}{c}\text { Paraplegia } \\
\qquad A B C^{12}\end{array}$} & \multicolumn{2}{|c|}{ All D's ${ }^{12}$} \\
\hline & $\mathrm{n}$ & $\%$ & $\mathrm{n}$ & $\%$ & $\mathrm{n}$ & $\%$ \\
\hline Physical stimulation & 44 & 68 & 87 & 80 & 11 & 23 \\
\hline Chemical stimulation & 15 & 23 & 11 & 10 & 7 & 15 \\
\hline Abdominal pressure & 4 & 6 & 6 & 6 & 3 & 6 \\
\hline Near normal & 2 & 3 & 5 & 5 & 26 & 55 \\
\hline Total & 65 & 100 & 109 & 100 & 47 & 100 \\
\hline
\end{tabular}

Table 3 Physician diagnosis of various complications by GI management technique

\begin{tabular}{|c|c|c|c|c|c|c|c|c|}
\hline & \multicolumn{8}{|c|}{ Percent with diagnosis } \\
\hline & \multicolumn{2}{|c|}{ Hemorrhoids } & \multicolumn{2}{|c|}{$\begin{array}{l}\text { Rectal } \\
\text { abscess }\end{array}$} & \multicolumn{2}{|c|}{$\begin{array}{c}\text { Fecal } \\
\text { incontinence }\end{array}$} & \multicolumn{2}{|c|}{ Constipation } \\
\hline & $\mathrm{n}$ & $\%$ & $\mathrm{n}$ & $\%$ & $\mathrm{n}$ & $\%$ & $\mathrm{n}$ & $\%$ \\
\hline Physical stimulation $(n=142)$ & 14 & 9.9 & 9 & 6.3 & 12 & 8.5 & 20 & 14.1 \\
\hline Chemical stimulation $(n=33)$ & 7 & 21.2 & 3 & 9.1 & 1 & 3.0 & 3 & 9.1 \\
\hline Abdominal pressure $(n=13)$ & 1 & 7.7 & 3 & 23.1 & 1 & 7.7 & 2 & 15.4 \\
\hline Near normal $(n=33)$ & 2 & 6.1 & 1 & 3.0 & 0 & 0 & 1 & 3.0 \\
\hline Total $(n=221)$ & 24 & 10.9 & 16 & 7.2 & 14 & 6.3 & 26 & 11.8 \\
\hline
\end{tabular}

*Statistically significant differences in outcome $(P<0.05)$

Table 4 Physician diagnosis of various complications by neurologic grouping

\begin{tabular}{|c|c|c|c|c|c|c|c|c|}
\hline & & & & recent & iagn & & & \\
\hline & & oids & & & & & & tion \\
\hline & $\mathrm{n}$ & $\%$ & $\mathrm{n}$ & $\%$ & $\mathrm{n}$ & $\%$ & $\mathrm{n}$ & $\%$ \\
\hline Tetraplegia $\mathrm{ABC}^{12}(n=65)$ & 7 & 10.8 & 6 & 9.2 & 9 & 13.8 & 3 & 4.6 \\
\hline Paraplegia $\mathrm{ABC}^{12}(n=109)$ & 15 & 13.8 & 9 & 8.3 & 4 & 3.7 & 21 & 19.3 \\
\hline All $\operatorname{Ds}^{12}(n=47)$ & 2 & 4.3 & 1 & 2.1 & 1 & 2.1 & 2 & 4.3 \\
\hline Total $(n=221)$ & 24 & 10.9 & 17 & 7.2 & 14 & 6.3 & 26 & 11.8 \\
\hline
\end{tabular}

*Statistically significant differences in outcome $(P<0.05)$

Table 5 Self-reports of various complications by bowel management technique

\begin{tabular}{lcccccr}
\hline & \multicolumn{2}{c}{ Constipation } & \multicolumn{2}{c}{ Bowel accidents } & \multicolumn{2}{c}{ GI pain } \\
& $\mathrm{n}$ & $\%$ & $\mathrm{n}$ & $\%$ & $\mathrm{n}$ & $\%$ \\
\hline Physical stimulation $(n=142)$ & 63 & 44.4 & 37 & 26.1 & 59 & 41.5 \\
Chemical stimulation $(n=33)$ & 12 & 36.4 & 12 & 36.4 & 6 & 18.2 \\
Abdominal pressure $(n=13)$ & 4 & 30.8 & 5 & 38.5 & 2 & 15.4 \\
Near normal $(n=33)$ & 13 & 39.4 & 6 & 18.2 & 10 & 30.3 \\
Total $(n=221)$ & 92 & 41.6 & 60 & 27.1 & 77 & 34.8 \\
\hline
\end{tabular}

*Statistically significant differences in outcome $(P<0.05)$ 
Table 6 Self-reports of various complications by neurological group

\begin{tabular}{lcccccc}
\hline & \multicolumn{2}{c}{ Constipation } & \multicolumn{2}{c}{ Bowel accidents } & \multicolumn{2}{c}{ GI pain } \\
& $\mathrm{n}$ & $\%$ & $\mathrm{n}$ & $\%$ & $\mathrm{n}$ & $\%$ \\
\hline Tetraplegia $\mathrm{ABC}^{12}(n=65)$ & 29 & 44.6 & 19 & 29.0 & 20 & 30.8 \\
Paraplegia $\mathrm{ABC}^{12}(n=109)$ & 44 & 40.4 & 30 & 27.5 & 46 & 42.2 \\
All Ds ${ }^{12}(n=47)$ & 19 & 40.4 & 11 & 23.4 & 11 & 23.4 \\
Total $(n=221)$ & 92 & 41.6 & 60 & 27.1 & 77 & 34.8 \\
\hline
\end{tabular}

divided at T9-the approximate level below which sufficient abdominal muscle function for generating a Valsalva maneuver might be expected-those with injuries at or above $\mathrm{T} 9$ did not differ from those with injuries below T9 with repect to complications.

Age and years post injury were not significantly associated in a logistic regression model with any of the self-reported outcomes, diagnoses of abscesses, and diagnoses of diarrhea. Increasing age was associated with increased likelihood of being diagnosed with hemorrhoids $(P=0.015)$; each decade of age increased the odds of being diagnosed with hemorrhoids 1.74 times. While the duration of injury did not have a similar effect on hemorrhoids, it was associated with an increased likelihood of being diagnosed with constipation $(P=0.005)$; each decade post-injury increased the odds of a diagnosis of constipation 2.31 times. Despite the association of age and time since injury $(r=0.554)$, age was not similarly associated with constipation.

\section{Discussion}

These data indicate a high prevalence of undesirable bowel symptoms in an aging SCI population and further demonstrate some of the effects that both age and duration of injury have on these symptoms. Perhaps somewhat surprising is the non-significant yet counter-intuitive finding with respect to hemorrhoids. Though many clinicians have suggested that hemorrhoids are related to repeated mechanical trauma, those with chemically-induced bowel management programs reported a greater incidence of this complication. Unfortunately, in what may be an important limitation to this study, the extent to which study participants currently using one form of bowel management might previously have used other methods-particularly manual stimulation-could not be addressed. Similarly, the potential impact on complications of other methods of evacuation that individuals might use or have used in addition to their primary method also could not be evaluated. Moreover, the extent to which these long-injured survivors may be using bowel management techniques inconsistent with or at least not representative of current rehabilitation practices was not addressed.

Also noteworthy, but not readily explainable, is the higher incidence of rectal abscesses among those utilizing the Valsalva maneuver to manage their bowels. Although the group's small size precluded tests for statistical significance, further examination of the outcomes of Valsalva users may be indicated. Finally, seeming relationships between diarrhea and constipation and neurological grouping and bowel management technique suggests the need for additional study targeting the identification of risk factors for these two complictions.

The difference between incidence of complications as recorded by the physician and as reported by the patient is noteworthy. These differences, where physicians appear to record fewer complications, may reflect patients' tendency to report the full spectrum of mild to moderate problems to a researcher, but only the most severe problems to their physicians. Another possible explanation may be a lack of physician documentation unless aggressive medical intervention is needed. In any case, not only is the significance of these differences unclear, but it also is not known whether they are equally present in more newly-injured patients who work closely with an entire rehabilitation team and who begin learning self-advocacy skills very early.

In addition to amassing more detailed data pertaining to dietary habits, future research with this study population will add three new, potentially relevant variables: body position during the bowel program, duration of the bowel program, and the impact of bowel function on activity. An upright position, while helpful to effective evacuation, may result in increased hydrostatic pressure in the perirectal blood vessels, thereby increasing the likelihood of hemorrhoids; the length of time to complete the bowel program may have a similar effect. Thus 5 min of Valsalva maneuvers in the upright position could result in a smaller increase in hydrostatic pressure than the same maneuvers performed for an hour and a half in a recumbent position. Finally, the impact of bowel management on the organization of an individual's life will be examined. The extent to which individuals must live their lives around their bowels-and the impact that the bowel program has on lifestyle-will be studied.

\section{Conclusion}

This study confirms clinical observations that bowel dysfunction and complaints of bowel problems are prevalent among individuals with long-term spinal cord 
injuries. Pathology varies by type of bowel management, which itself varies with the type of neurologic injury. What remains unanswered is whether increased surveillance and earlier intervention by dietary counselling, medications, and/or changes in bowel management can decrease or modify the extent of these undesirable outcomes.

\section{Acknowledgements}

Funding support for this research was provided by a Demonstration and Epidemiology award for the Prevention of Secondary Disabilities from the US Centers for Disease Control and Prevention (award No.R04/ CCR808517), and by a Rehabilitation Research and Training Center grant from the US Department of Education's National Institute on Disability and Rehabilitation Research, for the study of Aging with Spinal Cord Injury (award No.H133B30040).

\section{References}

1 Cosman BC, Stone JM, Perkash I. Gastrointestinal complications of chronic spinal cord injury. J Amer Paraplegia Soc 1991; 14: $175-181$

2 Longo WE, Ballantyne GH, Modlin IM. The colon, anorectum, and spinal cord patient: A review of the functional alterations of the denervated hindgut. Dis Colon Rectum 1989; 32: $261-267$.
3 Menardo G et al. Large-bowel transit in paraplegic patients. Dis Colon Rectum 1987; 30: $924-928$.

4 Fealey RD, Szurszewski JH, Merritt JL, DiMagno EP. Effect of traumatic spinal cord transection on human upper gastrointestinal motility and gastric emptying. Gastroenterology 1984; 87: $69-75$.

5 Glick ME et al. Colonic dysfunction in patients with thoracic spinal cord injury. Gastroenterology 1984; 86: 287-294.

6 Stone JM, Nino-Murcia M, Wolfe VA, Perkash I. Chronic gastrointestinal problems in spinal cord injury patients: A prospective analysis. Am J Gastroenterology 1990; 85: $1114-$ 1119.

7 Hawkins RL, Bailey HR, Donovan WH. Autonomic dysreflexia resulting from prolapsed hemorrhoids: Report of a case. Dis Colon Rectum 1994; 37: 492 - 493.

8 Apstein MD, Dalecki-Chipperfield K. Spinal cord injury is a risk factor for gallstone disease. Gastroenterology 1987; 92: 966-968.

9 Longo WE, Ballantyne GH, Modlin IM. Colorectal disease in spinal cord patients: An occult diagnosis. Dis Colon Rectum 1990; 33: $131-134$

10 Frankel HL. Bowel training. Paraplegia 1967; 4: 254-258.

11 Connell AM. The physiology and pathophysiology of constipation. Paraplegia 1967; 4: 244-250.

12 Dittuno JF, Young W, Donovan WH, Creasey G. The international standards booklet for neurological and functional classification of spinal cord injury. Paraplegia 1994; 32: 70-80.

13 Menter RR, Hudson LM. Effects of age at injury and the aging process. In: Spinal Cord Injury: Clinical Outcomes from the Model Systems. Stover SL, DeLisa JA, Whiteneck GG eds. Gaithersburg, MD: Aspen Publications, 1995.

14 Whiteneck GG et al. Mortality, morbidity and psychosocial outcomes of persons spinal cord injured more than 20 years ago. Paraplegia 1992; 30: 617-630. 